\title{
Lobulación Pulmonar De La Rata Blanca (Rattus norvegicus albinus)
}

\author{
Pulmonary Lobulation of the White Rat (Rattus norvegicus albinus)
}

\author{
Ricardo Olivares P-M.* \& Julio Gil G.**
}

OLIVARES, P-M. R. \& GIL, G. J. Lobulación pulmonar de la rata blanca (Rattus norvegicus albinus). Int. J. Morphol., 34(3):901-903, 2016.

RESUMEN: La rata de laboratorio (Rattus norvegicus albinus), ha sido usada como modelo biológico para investigación, desde hace mucho tiempo. Por lo cual conocer su anatomía, en particular lo que dice relación con la lobulación pulmonar, resulta de utilidad para eventuales aplicaciones en las ciencias biomédicas. Para tal propósito, se utilizaron 10 ratas machos de la cepa Wistar, las que luego de ser sacrificadas por sobredosis de tiopental sódico intraperitoneal y antes de ser extraídos los pulmones de la cavidad torácica, a 7 de ellas, se les inyectó formalina al $10 \%$ intratraqueal y a las otras 3, acrílico dental autocurable, para posteriormente, colocar el tejido en una solución $\mathrm{KOH}$, para su corrosión. Es así como, de acuerdo a la arborización bronquial observada, se evidenció en el pulmón derecho 4 lóbulos (craneal, medio, caudal y accesorio) y 1 sólo lóbulo no dividido en el pulmón izquierdo.

PALABRAS CLAVE: Pulmón; Lobulación pulmonar; Rata; Modelo animal; Corrosión.

\section{INTRODUCCIÓN}

La rata de laboratorio (Rattus norvegicus albinus), ha sido usada como modelo biológico para investigación, desde hace mucho tiempo. En el año 1999 2,6 millones de ratas ( $27 \%$ de los animales usados), fueron utilizados en la Comunidad Europea (Möller \& Vásquez, 2011). Por lo cual conocer su anatomía, en particular lo que dice relación con la lobulación pulmonar, resulta de utilidad para eventuales aplicaciones en las ciencias biomédicas.

Los pulmones de los vertebrados están diseñados para la respiración aérea, siendo generalmente pares. Embriológicamente los pulmones corresponden a evaginaciones endodérmicas ventrales del tubo digestivo, comunicándose con el exterior a través de la tráquea (Kardong, 2005).

El pulmón tiene la estructura de un sistema de conductos compuesto de túbulos y alvéolos. Los dos bronquios principales se dividen en bronquios lobulares, cuya conformación difiere en el pulmón derecho e izquierdo según los diferentes mamíferos domésticos. La ramificación de los bronquios hasta los alvéolos pulmonares terminales puede realizarse en forma dicotómica o tricotómica dando lugar a la formación del árbol bronquial (König \& Liebich, 2005).
De hecho ratas y otros roedores, presentan una ramificación más monopodal que dicotómica (Yeh et al., 1979).

Las subdivisiones del árbol bronquial constituyen el fundamento de la denominación de los lóbulos pulmonares, cuya diferenciación varía según la especie animal (König \& Liebich).

\section{MATERIAL Y MÉTODO}

Se utilizaron 10 ratas machos de la cepa Wistar de un peso promedio de $355 \pm 26 \mathrm{~g}$, provenientes del Bioterio del Instituto de Nutrición y Tecnología de los AlimentosUniversidad de Chile, criadas en condiciones acordes a la Committee for the Update of the Guide for the Care and Use of Laboratory Animals (2010), las que fueron disecadas en fresco, luego de ser sacrificadas por sobredosis de tiopental sódico intraperitoneal (AVMA, 2013). Antes de ser extraídos los pulmones de la cavidad torácica, a 7 de ellas, se les inyectó vía intratraqueal parte cervical, formalina al $10 \%$ y a las otras 3 , acrílico dental autocurable, para posteriormente, colocar el tejido en una solución al $25 \%$ de $\mathrm{KOH}$,

\footnotetext{
* Departamento de Patología Animal. Facultad de Ciencias Veterinarias y Pecuarias, Universidad de Chile, Santiago, Chile.

** Departamento de Anatomía, Embriología y Genética Animal. Facultad de Veterinaria, Universidad de Zaragoza, Zaragoza, España.
} 


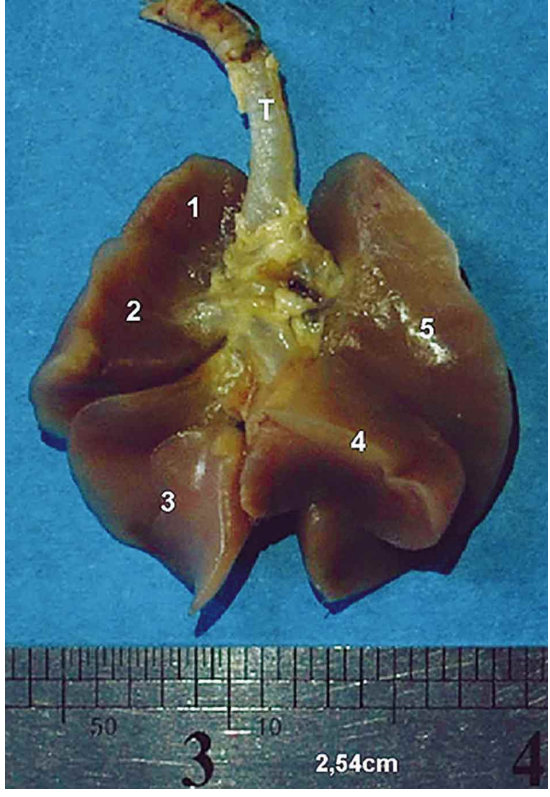

Fig. 1. Lobulación pulmonar de rata. 1) Lóbulo Craneal Pulmón Derecho (PD), 2) Lóbulo Medio PD, 3) Lóbulo Caudal PD, 4) Lóbulo Accesorio PD, 5) Pulmón Izquierdo, y T= Tráquea (medidas en pulgadas).

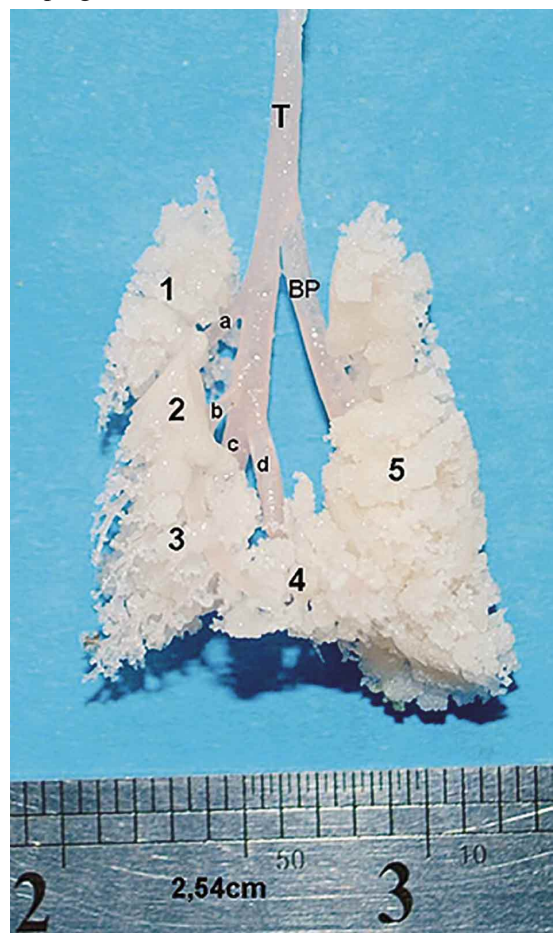

Fig. 2. Arbol bronquial de rata con acrílico autocurable. 1) Lóbulo Craneal Pulmón Derecho (PD), 2) Lóbulo Medio PD, 3) Lóbulo Caudal PD, 4) Lóbulo Accesorio PD, 5) Pulmón Izquierdo, $\mathrm{T}=$ Tráquea, $\mathrm{BP}=$ Bronquio Principal, a) Bronquio lobar del lóbulo craneal PD, b) Bronquio lobar del lóbulo medio PD, c) Bronquio lobar del lóbulo caudal PD, y d) Bronquio lobar del accesorio $\mathrm{PD}$ (medidas en pulgadas). para su corrosión (Canals et al., 2005). El resto de los pulmones se conservaron por inmersión en formalina al $5 \%$, hasta su descripción anatómica.

\section{RESULTADOS}

De acuerdo a la arborización bronquial observada, se evidenció en el pulmón derecho 4 lóbulos (craneal, medio, caudal y accesorio) y 1 sólo lóbulo no dividido en el pulmón izquierdo (Figs. 1 y 2).

\section{DISCUSIÓN}

La lobulación pulmonar observada (Fig. 1), coincide con lo descrito por Suckow et al. (1975), quedando verificado al haber utilizado la técnica de corrosión, que permite una mejor visualización de la arborización bronquial (Fig. 2), la cual determina la cantidad de lóbulos presentes en cada pulmón (König \& Liebich).

El hallazgo de 4 lóbulos en el pulmón derecho coincide con lo observado en rumiantes, carnívoros y suidos, encontrándose en los primeros el lóbulo craneal dividido en 2 partes (craneal y caudal). Los équidos sólo presentan 3 lóbulos (craneal, caudal y accesorio). Con respecto al pulmón izquierdo, éste se encuentra dividido en 2 lóbulos (craneal y caudal), estando el lóbulo craneal, a excepción de los équidos, dividido en dos partes (craneal y caudal) (Schaller, 1996; International Committee on Veterinary Gross Anatomical Nomenclature, 2005).

Además, el lóbulo accesorio en el pulmón derecho, es una característica común a muchos mamíferos no relacionados, por lo que parece ser una característica plesiomórfica en la clase Mammalia (Nakakuki, 1980).

Llama la atención la presencia de sólo 1 lóbulo no dividido en el pulmón izquierdo de la rata. Lo anterior ha sido corroborado en la Rata Gigante Africana (Cricetomis gambianus), que se considera una especie representativa de la familia Muridae, la cual constituye un grupo del orden Rodentia (Perry et al., 2006). En dicho estudio se disecaron 20 ejemplares adultos de dicha especie (11 machos y 9 hembras), observándose un pulmón izquierdo no lobulado y un pulmón derecho con los 4 lóbulos ya descritos (Ibe et al., 2011).

El conocer la lobulación pulmonar de la rata de laboratorio, resulta de utilidad para eventuales aplicaciones en las ciencias biomédicas, como lo es por ejemplo en la cirugía experimental resectiva pulmonar (Martínez et al., 2014). Dicho procedimiento quirúrgico, constituye el tratamiento de múltiples patologías benignas y malignas humanas, tanto en niños como en adultos, ya que se ha visto que el pulmón restablece su masa, estructura y función posterior a estas cirugías, lo que se denomina, crecimiento compensatorio. Este desarrollo compensatorio se ha estudiado en diferentes animales, en los que la rata es quien muestra una asombrosa similitud con los humanos, tanto en la anatomía, como en el desarrollo funcional pulmonar (Burri, 2006).

AGRADECIMIENTOS: Luis Nibaldo Avila Muñoz, Técnico Bioterio INTA, U. de Chile. 
OLIVARES, P-M. R. \& GIL, G. J. Pulmonary lobulation of the white rat (Rattus norvegicus albinus). Int. J. Morphol., 34(2):901-903, 2016.

SUMMARY: The laboratory rat (Rattus norvegicus albinus) laboratory rat has long been used as a biological model for research. Therefore knowledge of its anatomy, particularly as related to pulmonary lobulation is useful for any applications in the biomedical sciences. For this reason, 10 male rats of the Wistar strain were used. After being sacrificed by overdose of intraperitoneal thiopental and before extracting the lungs from the thoracic cavity, 7 of them were injected $10 \%$ formalin intratracheal and the other 3 , self-curing dental acrylic, subsequently placing the tissue in a $\mathrm{KOH}$ solution for corrosion. Thus, according to the observed bronchial tree, 4 lobes were observed in the right lung (cranial, middle, caudal and accessory) and only one lobe not divided in the left lung.

KEY WORDS: Lung; Pulmonary lobulation; Rat; Animal model; Corrosion.

\section{REFERENCIAS BIBLIOGRÁFICAS}

American Veterinary Medical Association (AVMA). AVMA Guidlines for the Euthanasia of Animals: 2013 Edition. Schaumburg, American Veterinary Medical Association, 2013.

Burri, P. H. Structural aspects of postnatal lung development alveolar formation and growth. Biol. Neonate, 89(4):313-22, 2006.

Canals, M.; Olivares, R. \& Rosenmann, M. A radiographic method to estimate lung volume and its use in small mammals. Biol. Res., 38(1):41-7, 2005

Committee for the Update of the Guide for the Care and Use of Laboratory Animals. Guide for the Care and Use of Laboratory Animals. $8^{\text {th }}$ ed. Washington D. C., The National Academies Press, 2010.

Ibe, C. S.; Salami, S. O. \& Onyeanusi, B. I. Macroscopic anatomy of the lower respiratory system in a nocturnal burrowing rodent: African giant pouched rat (Cricetomys gambianus, Waterhouse 1840). Anat. Histol. Embryol., 40(2):112-9, 2011.

International Committee on Veterinary Gross Anatomical Nomenclature (I. C. V. G. A. N.). Nomina Anatomica Veterinaria $(N A V) .5^{\text {th }}$ ed. Knoxville, International Committee on Veterinary Gross Anatomical Nomenclature \& General Assembly of the World Association of Veterinary Anatomists (W.A.V.A.), 2005.

Kardong, K. V. Vertebrados: Anatomía Comparada, Función y Evolución. $2^{\mathrm{a}}$ ed. Madrid, McGraw-Hill Interamericana, 2005.

König, H. E. \& Liebich, H. G. Anatomía de los Animales Domésticos: Texto y Atlas en Color. $2^{\mathrm{a}}$ ed. Buenos Aires, Editorial Médica Panamericana, 2005.

Martínez, M. M.; Velázquez, S. N.; Flores, A. E.; Navarrete, H. A.; Zúñiga, H. F.; Zitko, M. P.; García, B. C.; González, B. S. \& Zúñiga, R. S. Lobectomía en ratas. Modelo quirúrgico experimental. Resultados en la densidad alveolar. Rev. Chil. Cir., 66(2):127-33, 2014.
Möller, B. R. \& Vázquez, O. N. Anatomy of the Liver in Wistar Rat (Rattus norvegicus). Int. J. Morphol., 29(1):76-9, 2011.

Nakakuki, S. Comparative Anatomical Studies on the Mammalian Lung. Tokyo, Tokyo Noko Daigaku Nogakubu Gakujutsu Hokoku, 1980.

Perry, N. D.; Hanson, B.; Hobgood, W.; Lopez, R. L.; Okraska, C. R.; Karem, K.; Damon, I. K. \& Carroll, D. S. New invasive species in Southern Florida: Gambian Rat (Cricetomys gambianus). J. Mammal., 87(2):262-4, 2006.

Shaller, O. Nomenclatura Anatómica Veterinaria Ilustrada. Zaragoza, Acribia Editorial, 1996.

Suckow, M. A.; Weisbroth, S. H. \& Franklin, C. L. The Laboratory Rat. London, Academic Press, 1975. pp.106-8.

Yeh, H. C.; Schum, G. M. \& Duggan, M. T. Anatomic models of the traqueobronquial and pulmonary regions of the rat. Anat. Rec., 195(3):483-92, 1979.



Email: rolivare@uchile.cl

Recibido : 08-07-2015

Aceptado: 15-04-2016 